\title{
THE INTEGRAL BURST ALERT SYSTEM
}

\author{
S. Mereghetti ${ }^{1}$, D.I. Cremonesi ${ }^{1}$, and J. Borkowski ${ }^{2}$ \\ ${ }^{1}$ Istituto di Fisica Cosmica "G.Occhialini" - CNR, Milano, Italy \\ ${ }^{2}$ INTEGRAL Science Data Center, Versoix, Switzerland
}

\begin{abstract}
We describe the INTEGRAL Burst Alert System (IBAS): the automatic software developed at the INTEGRAL Science Data Center to allow the rapid distribution of the coordinates of the Gamma-Ray Bursts detected by INTEGRAL.

IBAS is implemented as a ground based system, working on the near-real time telemetry stream. It is expected that the system will detect more than one GRB per month in the field of view of the main instruments. Positions with an accuracy of a few arcminutes will be distributed to the community for follow-up observations within a few tens of seconds of the event. The system will also upload commands to optimize the possible detection of bursts in the visible band with the INTEGRAL Optical Monitor Camera.
\end{abstract}

Key words: Paper presented at the IV INTEGRAL Workshop, Alicante 4-8 September 2001.

\section{INTRODUCTION}

The origin of gamma-ray bursts (GRB's) remained one of the great mysteries of high energy astrophysics for more than 25 years after the publication of their discovery (Klebesadel et al. 1973). The puzzle became even more complicated after the first results obtained with the BATSE instrument. This detector, especially designed to significantly increase the GRB sample, led to the unexpected result of a noneuclidean LogN-LogS coupled with a uniform angular distribution in the sky (see, e.g., Fishman 1995, and references therein). It soon became clear that the only way to make a significant advance in the field was the possibility of identifying the counterparts of GRB at other wavelengths.

The instruments on board INTEGRAL, though not specifically optimized to observe GRB's, offer the possibility of rapidly obtaining accurate positions of the GRB's observed by chance in their large field of view. It was therefore proposed to implement a "burst alert system" in order to allow rapid multiwavelength follow-ups (Pedersen et al. 1997). Such a proposal was boosted by the exciting results obtained with the BeppoSAX satellite (Costa et al. 1997, van Paradijs et al. 1997), that clearly demonstrated the capabilities of a coded mask instrument, similar to the INTEGRAL ones, in quickly localizing GRB's.

\section{OVERVIEW OF THE INTEGRAL BURST ALERT SYSTEM (IBAS)}

Since no on-board GRB detection system is foreseen on INTEGRAL, the search for GRB's will be performed on ground by means of a near real time analysis running at the INTEGRAL Science Data Center (ISDC). The telemetry data, received at the MOC, will be transmitted on a $128 \mathrm{kbs}$ dedicated line to the ISDC. Here the relevant data packets will be extracted and immediately fed into a dedicated software system called IBAS (Integral Burst Alert System), independent from the main data processing pipeline of the ISDC (Mereghetti et al. 1999).

It is expected that ISGRI, the first layer of the IBIS instrument, operating in the $15-300 \mathrm{keV}$ energy range, will yield the best chances to detect a large number of GRB's and to accurately determine their positions. The SPI instrument, with a similar sensitivity and large field of view, can also detect GRB's, but its angular resolution is not as good as the IBIS one. Relatively strong bursts in the central part of the field of view will be detectable by SPI and IBIS, therefore a simultaneous trigger in both instruments could be used to increase the confidence in the reality of the event. However, due to the different sensitivity curves as a function of energy and source direction, it is likely that several GRB's will trigger only in a single detector. For this reason the IBAS validation process will not require a multiple detection to confirm the occurrence of a GRB.

The first step of the GRB search will be based on a simple monitoring of the incoming count rates, without resorting to more complex image analysis. In practice this will be done by looking for significant excesses with respect to a running average, in a way 
similar to traditional on-board triggering algorithms. In fact any transient source strong enough to appear as a significant new peak in the deconvolved images will also produce a detectable excess in the overall count rate. The search will be simultaneously performed in many different time scales and energy ranges, to optimize the sensitivity to GRB's with different characteristics.

When a candidate event is detected, a process of image analysis shall start to verify the origin of the count rate variation and to ensure that the event was not caused by an instrumental malfunctioning (e.g. a bad detector pixel) or by a background variation (see Figures 1, 2, 3). Images shall be accumulated for different time intervals, deconvolved with very fast algorithms, and compared to the pre-burst reference images in order to detect the appearance of the GRB as a new source. This last step is of fundamental importance, since in general different sources will be present in the field of view. The image analysis will be based on the time intervals, derived from the GRB light curve, that optimize the signal to noise ratio.

If the event is genuine, the satellite attitude information will be applied to derive a sky position that is then automatically transmitted, by e-mail and/or direct TCP/IP socket, to all the subscribed users. In addition, if the GRB is located in the sky region covered by the OMC, an appropriate telecommand will be generated and sent to the satellite to reconfigure its observing parameters (see Section 6). Because full event validation and localization might require a longer time, we foresee different levels of alert messages providing increasingly accurate and reliable information. These messages will be configured in such a way to allow an easy filtering by the users in order to react only to the situations that best fit their needs.

\section{NUMBER OF EXPECTED GRB'S}

A very simple estimate of the number of GRB's expected within the field of view of the INTEGRAL instruments can be done by scaling the total rate of events measured by BATSE ( 666 GRB year ${ }^{-1}$, Paciesas et al. 1999). For the field of view of IBIS $\left(\sim 30^{\circ} \times 30^{\circ}\right)$, this yields $666 \times(0.23$ sterad $/ 4 \pi) \sim$ 12 GRB year ${ }^{-1}$.

A more accurate estimate must take into account the varying sensitivity within the IBIS field of view and the different energy range of the BATSE detectors. This can be done by convolving the IBIS sensitivity and solid angle as a function of off-axis angle with the BATSE LogN-LogP relations, converted to the appropriate energy range assuming an average GRB spectral shape. It turns out that such computations do not significantly change the above rough result. In fact we obtain expected rates of $\sim 13,10$ and 8 GRB year $^{-1}$, within the ISGRI field of view, adopting respectively the LogN-LogP corresponding to BATSE

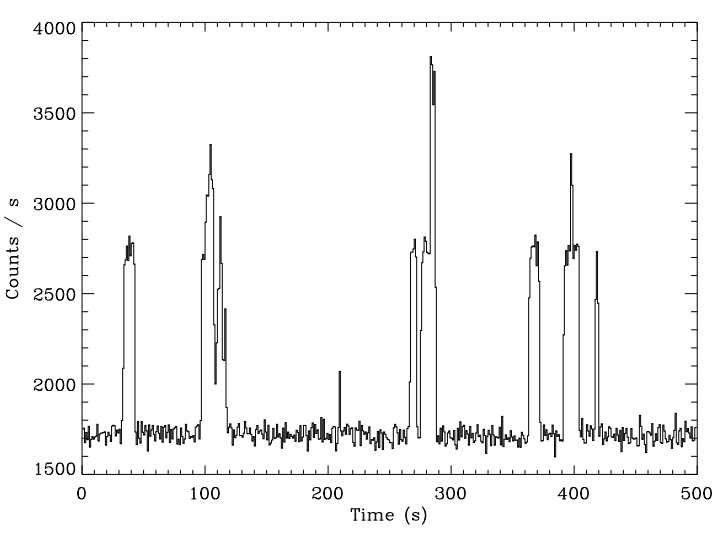

Figure 1. Example of simulated ISGRI data containing a GRB and several noisy pixels. The figure shows the light curve of the overall count rate on the instrument, including the background ( 1200 $\left.\mathrm{cts} \mathrm{s}^{-1}\right)$, a source with intensity of $1 \mathrm{Crab}\left(\sim 530 \mathrm{cts} \mathrm{s}^{-1}\right)$, a GRB with equivalent peak flux in the 50-300 keV range of $\sim 0.7 \mathrm{cts} \mathrm{cm}^{-2} \mathrm{~s}^{-1}$, and several noisy pixels producing short spikes of high count rate.

trigger times $\Delta \mathrm{T}$ of $1 \mathrm{~s}, 256 \mathrm{~ms}$ and $64 \mathrm{~ms}$.

The major uncertainty on the derived GRB rates is related to the extrapolation of the BATSE LogN$\operatorname{LogP}$ curves down to the ISGRI sensitivity $(\sim 0.1$ ph $\mathrm{cm}^{-2} \mathrm{~s}^{-1}, 50-300 \mathrm{keV}$ peak flux for $\left.\Delta \mathrm{T}=1 \mathrm{~s}\right)$. Such an extrapolation depends on the poorly known spectral shape of very weak GRB's.

It must be noted that these estimates do not take into account the likely existence of GRB's with different characteristics than those observed by BATSE. For instance it is clear that BATSE had a limited sensitivity for events shorter than $64 \mathrm{~ms}$, as well as a strong bias against the detection of long, slowly rising GRB's. It it therefore very likely that the figures reported above will be an underestimate, and that a few events per month will be available through IBAS for rapid multi-wavelength follow-up observations.

\section{LOCATION ACCURACY}

The source location accuracy (SLA) of coded mask imaging systems depends on the signal to noise ratio of the source. For sources detected with a high statistical significance the SLA can be a small fraction of the angular resolution. Theoretical evaluations, confirmed by several independent simulations, have shown that for ISGRI a SLA smaller than 30" (90\% confidence level) can be obtained for a signal to noise ratio of 30 (see Figure 1). In such cases, the final accuracy on the GRB location is also affected by the uncertainties on the satellite attitude (see below). Of course, most of the detected GRB's will have relatively small signal to noise ratios, resulting in typical uncertainties, dominated by the photon 


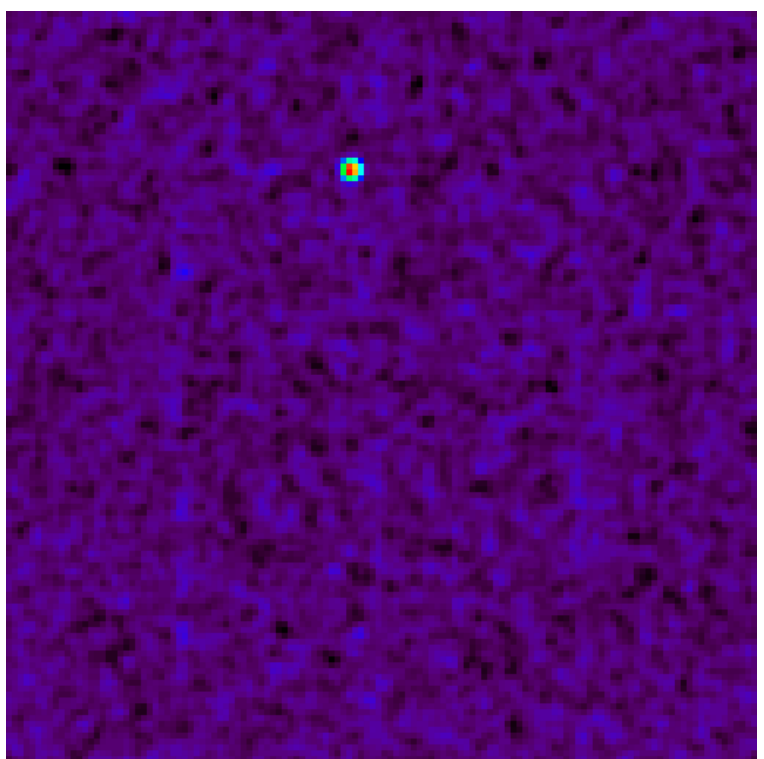

Figure 2. Image of the GRB event shown in Figure 1. The reference image of a time interval preceding the trigger has been subtracted, thus the GRB can be easily identified as the only peak in the deconvolved image.

statistics, of the order of $\sim 2-3^{\prime}$.

The expected INTEGRAL attitude accuracy depends on three cases: (1) attitude during slews, (2) attitude at the start of a stable pointing period, and (3) attitude from about ten minutes after the start to the end of a stable pointing period.

For case (1) a slew path simulator generates predictions of the pointing direction every 10 seconds. In most circumstances these predictions should be accurate to within $10^{\prime}(3 \sigma)$. The average slew rate during the Galactic Plane scans and during the normal dithering observations will be of the order of $\sim 1^{\circ} / \mathrm{min}$. Therefore, it will be possible to detect GRB's even during slews, although their location accuracy will be worse.

For case (2) the attitude is based upon a prediction of the expected position with respect to the previously commanded slew. In most cases (i.e. slews shorter than $2^{\circ}$ ) these predicted values will have an accuracy of the order of the star-tracker/instrument alignment uncertainty ( $30^{\prime \prime}$ or less). For larger slews the predicted attitude will have an error smaller than $5 \pm 1^{\prime}(3 \sigma)$.

Approximately 5 minutes after the start of a pointing (case 3) a "snapshot" attitude reconstruction will be completed based upon the first down-linked startracker map. The result shall be made available to the IBAS within a maximum delay of 10 minutes since the start of the stable pointing, yielding, as above, an attitude with accuracy $\lesssim 30^{\prime \prime}$.

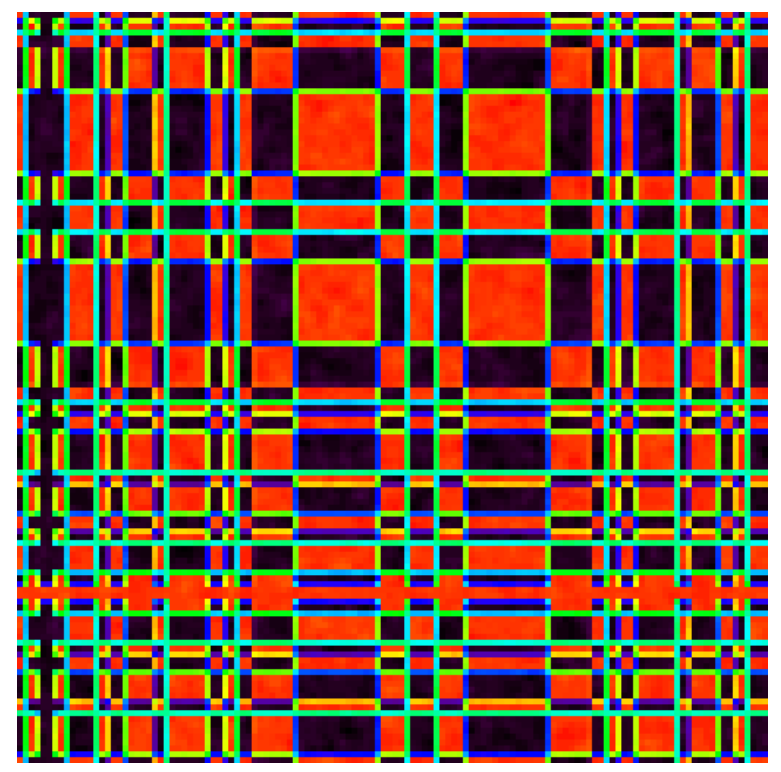

Figure 3. Deconvolved image corresponding to the time interval of one of the noisy pixel spikes shown in Figure 1. The image pattern, without a point source, clearly allows to reject this trigger.

\section{IBAS TIME PERFORMANCE}

Using simulated telemetry data, we have obtained the following performance figures for the current prototype version of IBAS running on a SUN ULTRA 10 workstation (440 MHz clock, 256 Mbytes RAM). Of course the final version will run on a faster workstation exclusively dedicated to the IBAS system.

The first IBAS steps (telemetry receipt, extraction and sorting of the relevant data packets, photon binning for the trigger search) require less than $0.1 \mathrm{~s}$.

The speed of the triggering algorithm depends on the duration of the smallest time interval considered and on the number of timescales. For example, sampling on bins of 1, 4, 16, 64 and $256 \mathrm{~ms}$, with a resolution of $1 \mathrm{~ms}$ it is still possible to process the telemetry at a speed twice faster than that of the real-time incoming data.

The most time consuming tasks are those related to the image analysis. For this reason, the first part of imaging after a trigger is based on a detection algorithm able to discover new sources by only considering (ghost) peaks in the fully coded field of view. This can be done within $\sim 200 \mathrm{~ms}$ for ISGRI and it allows to discriminate between false triggers and true events. Only when a likely point source is detected, a more thorough analysis will be done to locate the GRB. The deconvolution of the whole (totally plus partially coded) field of view and localization of the true source peak currently requires $\sim 5 \mathrm{~s}$ (we expect to improve this performance with a new optimized version of the code). 
Point Source Location Accuracy

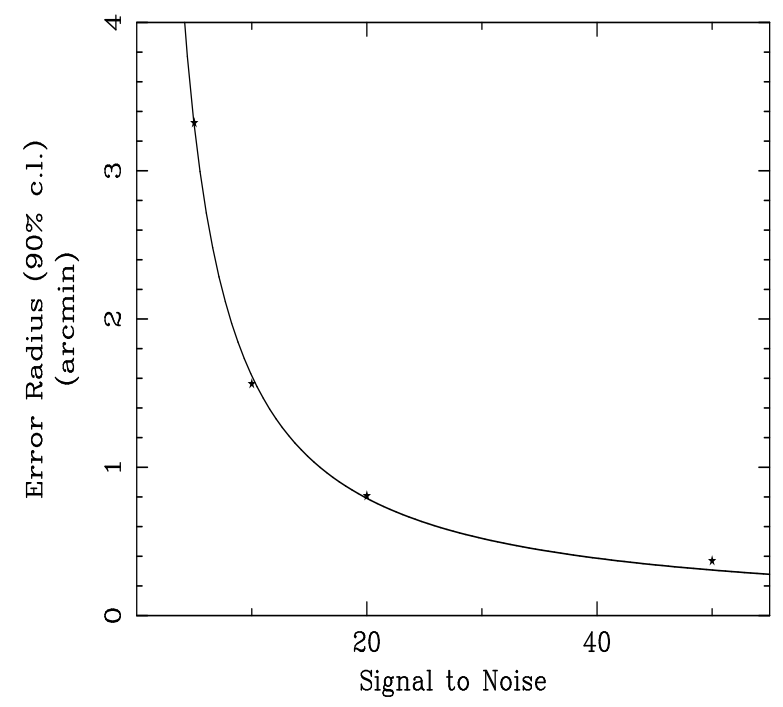

Figure 4. Source Location Accuracy as a function of the Signal to Noise ratio for the ISGRI detector.

alert within a few seconds after the trigger time. The delay between the trigger time and the GRB onset is of course dependent on the intensity and time profile of the event, but the IBAS simultaneous sampling in different timescales should ensure a minimum delay in most cases.

To this time budget one has to add the time required for the telemetry transmission from the satellite to the ISDC, that under normal circumstances should be smaller than $\sim 30 \mathrm{~s}$.

Thus, in many cases, we foresee to be able to generate first level alerts while the GRB is still ongoing.

\section{OPTICAL MONITOR RECOMANDING}

The Optical Monitor Camera (OMC) on board INTEGRAL has a field of view of $5^{\circ} \times 5^{\circ}$, but owing to the limited allocated telemetry $\left(\sim 1.7 \mathrm{kbit} \mathrm{s}^{-1}\right)$ only the data from a number of preselected windows around sources of interest will be downloaded during normal operations. IBAS will check whether the derived GRB position falls within the OMC field of view. In such a case, the appropriate telecommand with the definition of a new window centered on the interesting region will be generated and sent to the satellite. This will allow to quickly observe the GRB/afterglow emission in the optical band. The OMC observation should consist of many frames with short integration times to permit variability studies and to increase the sensitivity for very intense but short outbursts. The expected limiting magnitude is of the order of $\mathrm{V} \sim 14-15$ for an integration time of 10 $\mathrm{s}$, and $\mathrm{V} \sim 12-13$ for $1 \mathrm{~s}$.

\section{ACKNOWLEDGMENTS}

We are grateful to several people that have contributed to the IBAS project, including G.Bazzaro, S.Brandt, D.Jennings, R.Hudec, H.Pedersen, A.Pellizzoni, M.Pohl, T.Courvoisier and R.Walter. The IBAS development is supported by the Italian Space Agency. JB was supported by the Polish Committee for Scientific Research (KBN) under grant No. 2P03C00619p02.

\section{REFERENCES}

Costa E. et al., 1997, Nature 387, 783.

Fishman G.J., 1995, PASP 107, 1145.

Klebesadel R., Strong I., Olsen R., 1993, ApJ 182, L85.

Mereghetti S., et al. 1999, Proc. 3nd INTEGRAL Workshop - Astro. Lett. and Communications, 39, 301.

Paciesas W.S., et al., 1999, ApJSS 122, 465.

Pedersen H., Jennings D., Mereghetti S., Teegarden B., 1997, Proc. 2nd INTEGRAL Workshop ESA SP-382, 433.

van Paradijs J. et al., 1997, Nature 386, 686. 